\title{
An overview of comonotonicity and its applications in finance and insurance
}

\author{
Griselda Deelstra*
}

\author{
Jan Dhaene $^{\dagger}$
}

\author{
Michèle Vanmaele
}

May 25, 2010

\begin{abstract}
Over the last decade, it has been shown that the concept of comonotonicity is a helpful tool for solving several research and practical problems in the domain of finance and insurance. In this paper, we give an extensive bibliographic overview - without claiming to be complete - of the developments of the theory of comonotonicity and its applications, with an emphasis on the achievements over the last five years. These applications range from pricing and hedging of derivatives over risk management to life insurance.
\end{abstract}

\section{Comonotonicity}

Over the last two decades, researchers in economics, financial mathematics and actuarial science have introduced results related to the concept of comonotonicity in their respective fields of interest. In this paper, we give an overview of the relevant literature in these research fields, with the main emphasis on the development of the theory and its applications in finance and insurance over the last five years. Although it is our intention to give an extensive bibliographic overview, due to the high number of papers on applications of comonotonicity, it is impossible to present here an exhaustive overview of the recent literature. Further, we restrict this paper to a description of how and where comonotonicity comes in and refer to the relevant papers for a detailed mathematical description. In order to make this paper self-contained, we also provide a short overview of the basic definitions and initial main results of comonotonicity theory, hereby referring to part of the older literature on this topic.

The concept of comonotonicity is closely related to the following well-known result, which is usually attributed to both Hoeffding (1940) and Fréchet (1951): For any $n$-dimensional random vector $\underline{X} \equiv\left(X_{1}, X_{2}, \ldots, X_{n}\right)$ with multivariate cumulative distribution function (cdf) $F_{X}$ and marginal univariate cdf's $F_{X_{1}}, F_{X_{2}}, \ldots, F_{X_{n}}$ and for any $\underline{x} \equiv\left(x_{1}, x_{2}, \ldots, x_{n}\right) \in \mathbb{R}^{n}$ it holds that

$$
F_{\underline{X}}(\underline{x}) \leq \min \left(F_{X_{1}}\left(x_{1}\right), F_{X_{2}}\left(x_{2}\right), \ldots, F_{X_{n}}\left(x_{n}\right)\right) .
$$

In the sequel, the notation $R_{n}\left(F_{X_{1}}, F_{X_{2}}, \ldots, F_{X_{n}}\right)$ will be used to denote the class of all random vectors $\underline{Y} \equiv\left(Y_{1}, Y_{2}, \ldots, Y_{n}\right)$ with marginals $F_{Y_{i}}$ equal to the respective marginals $F_{X_{i}}$. The set $R_{n}\left(F_{X_{1}}, F_{X_{2}}, \ldots, F_{X_{n}}\right)$ is called the Fréchet class related to the random vector $\underline{X}$.

The upper bound in (1) is reachable in the Fréchet class $R_{n}\left(F_{X_{1}}, F_{X_{2}}, \ldots, F_{X_{n}}\right)$ in the sense that it is the cdf of an $n$-dimensional random vector with marginals given by $F_{X_{i}}, i=1,2, \ldots, n$.

\footnotetext{
${ }^{*}$ Department of Mathematics, ECARES, Université Libre de Bruxelles, CP 210, 1050 Brussels, Belgium

${ }^{\dagger}$ Faculty of Business and Economics, Katholieke Universiteit, 3000 Leuven, Belgium.

${ }^{\ddagger}$ Department of Applied Mathematics and Computer Science, Ghent University, Krijgslaan 281 S9, 9000 Gent, Belgium
} 
In order to prove the reachability property, consider a random variable $U$, uniformly distributed on the unit interval $(0,1)$. Then one has that

$$
\left(F_{X_{1}}^{-1}(U), F_{X_{2}}^{-1}(U), \ldots, F_{X_{n}}^{-1}(U)\right) \in R_{n}\left(F_{X_{1}}, F_{X_{2}}, \ldots, F_{X_{n}}\right),
$$

where the generalized inverses $F_{X_{i}}^{-1}$ are defined in the usual way:

$$
F_{X_{i}}^{-1}(p)=\inf \left\{x \in \mathbb{R} \mid F_{X_{i}}(x) \geq p\right\}, \quad p \in[0,1],
$$

with $\inf \emptyset=+\infty$, by convention. Furthermore,

$$
\operatorname{Pr}\left[F_{X_{1}}^{-1}(U) \leq x_{1}, F_{X_{2}}^{-1}(U) \leq x_{2}, \ldots, F_{X_{n}}^{-1}(U) \leq x_{n}\right]=\min \left(F_{X_{1}}\left(x_{1}\right), F_{X_{2}}\left(x_{2}\right), \ldots, F_{X_{n}}\left(x_{n}\right)\right),
$$

which holds for any $\underline{x} \in \mathbb{R}^{n}$. Throughout this paper, the notation $U$ will uniquely be used to denote a random variable which is uniformly distributed on the unit interval $(0,1)$.

The random vector $\left(F_{X_{1}}^{-1}(U), F_{X_{2}}^{-1}(U), \ldots, F_{X_{n}}^{-1}(U)\right)$ is said to have the comonotonic dependence structure. More generally, a random vector $\underline{X} \equiv\left(X_{1}, \ldots, X_{n}\right)$ is said to be comonotonic if

$$
F_{\underline{X}}(\underline{x})=\min \left(F_{X_{1}}\left(x_{1}\right), F_{X_{2}}\left(x_{2}\right), \ldots, F_{X_{n}}\left(x_{n}\right)\right), \quad \text { for any } \underline{x} \in \mathbb{R}^{n} .
$$

Other characterizations of comonotonicity can be found, e.g., in Denneberg (1994).

Furthermore, we will use the notation $\underline{X}^{c} \equiv\left(X_{1}^{c}, X_{2}^{c}, \ldots, X_{n}^{c}\right)$ to indicate a comonotonic random vector belonging to the Fréchet class $R_{n}\left(F_{X_{1}}, F_{X_{2}}, \ldots, F_{X_{n}}\right)$. The random vector $\underline{X}^{c}$ is often called a comonotonic counterpart or a comonotonic modification of $\underline{X}$. Obviously, one has that

$$
\underline{X}^{c} \stackrel{d}{=}\left(F_{X_{1}}^{-1}(U), F_{X_{2}}^{-1}(U), \ldots, F_{X_{n}}^{-1}(U)\right),
$$

where the notation $\stackrel{d}{=}$ is used to indicate 'equality in distribution'. The random vector $\underline{X}^{c}$ is said to have the comonotonic dependence structure or copula, see, e.g., Nelsen (1998).

The components of the comonotonic random vector $\left(F_{X_{1}}^{-1}(U), F_{X_{2}}^{-1}(U), \ldots, F_{X_{n}}^{-1}(U)\right)$ are maximally dependent in the sense that all of them are non-decreasing functions of the same random variable. Hence, comonotonic random variables are indeed 'common monotonic'. From an economic point of view this means that holding a long position (or a short position) in comonotonic random variables can never lead to a hedge, as the variability of one is never tempered by counter-variability of others.

Comonotonicity corresponds with the riskiest dependence structure observed in a given Fréchet space $R_{n}\left(F_{X_{1}}, F_{X_{2}}, \ldots, F_{X_{n}}\right)$. A natural question which arises is whether there exists also a least risky dependence structure in $R_{n}\left(F_{X_{1}}, F_{X_{2}}, \ldots, F_{X_{n}}\right)$. From Hoeffding (1940) and Fréchet (1951) it is known that the following bound holds in $R_{n}\left(F_{X_{1}}, F_{X_{2}}, \ldots, F_{X_{n}}\right)$ :

$$
F_{\underline{X}}(\underline{x}) \geq \max \left(\sum_{i=1}^{n} F_{X_{i}}\left(x_{i}\right)-n+1,0\right), \quad \text { for any } \underline{x} \in \mathbb{R}^{n} .
$$

It is straightforward to prove that $\left(F_{X_{1}}^{-1}(U), F_{X_{2}}^{-1}(1-U)\right) \in R_{2}\left(F_{X_{1}}, F_{X_{2}}\right)$ and that its cdf is given by $\min \left(F_{X_{1}}\left(x_{1}\right), F_{X_{2}}\left(x_{2}\right)\right)$. Hence, when $n=2$, the lower bound in (2) is reachable in $R_{2}\left(F_{X_{1}}, F_{X_{2}}\right)$ and the random couple $\left(F_{X_{1}}^{-1}(U), F_{X_{2}}^{-1}(1-U)\right)$ is said to have the countermonotonic dependence structure.

More generally, a bivariate random vector $\underline{X} \equiv\left(X_{1}, X_{2}\right)$ is said to be countermonotonic if

$$
F_{\underline{X}}(\underline{x})=\max \left(F_{X_{1}}\left(x_{1}\right)+F_{X_{2}}\left(x_{2}\right)-1,0\right), \quad \text { for any } \underline{x} \in \mathbb{R}^{n} .
$$


When $n \geq 3$, the lower bound in (2) is not always a cdf anymore and the concept of countermonotonicity cannot be generalized to higher dimensions without imposing additional conditions. Necessary and sufficient conditions for $\max \left(\sum_{i=1}^{n} F_{X_{i}}\left(x_{i}\right)-n+1,0\right)$ to be a cdf can be found, e.g., in Joe (1997).

Dhaene and Denuit (1999) consider Fréchet spaces containing non-negative mutually exclusive risks, that is risks that cannot be strictly positive together. They show that, under some reasonable assumptions, the Fréchet lower bound is reachable in such Fréchet classes and corresponds with the mutually exclusive risks of that space.

Embrechts et al. (2002) investigate the relation between comonotonicity and extremal correlations. They point out that a positive perfectly correlated random couple is comonotonic, whereas the inverse does not necessarily holds. Denuit and Dhaene (2003) investigate the relation between comonotonicity, respectively countermonotonicity, and several classical measures of association such as Pearson's correlation coefficient, Kendall's $\tau$, Spearman's $\rho$ and Gini's $\gamma$.

Thanks to the works of Schmeidler (1986), Roëll (1987) and Yaari (1987), comonotonicity has become an important concept in economic theories of decision under risk and uncertainty. Yaari developed a theory of risk dual to the classical expected utility theory of von Neumann and Morgenstern (1947) by modifying the independence axiom in the latter theory. In Yaari's theory, the concept of 'distorted expectations' arises as the equivalent of 'expected utilities' in von Neumann and Morgenstern's theory. These distorted expectations are additive for comonotonic random variables.

\section{Convex bounds for sums of random variables}

In risk theory and finance, one is often interested in the distribution of the sum $S=X_{1}+$ $X_{2}+\cdots+X_{n}$ of individual risks of a portfolio $\underline{X} \equiv\left(X_{1}, X_{2}, \ldots, X_{n}\right)$. Departing from the results of Hoeffding (1940) and Fréchet (1951), stochastic order bounds have been derived for sums $S$ of which the cdf's of the $X_{i}$ are known, but the joint distribution of the random vector $\left(X_{1}, X_{2}, \ldots, X_{n}\right)$ is either unspecified or too cumbersome to work with. Assuming that only the marginal distributions of the random variables are given (or used), the largest sum in convex order will occur when the random variables are comonotonic.

In this section, we give a short overview of these stochastic ordering results. Early references to part of the ideas and results presented below are Meilijson and Nadas (1979), Tchen (1980) and Rüschendorf (1983). For proofs and more details on the presented results, we refer to the overview paper of Dhaene et al. (2002a). An overview of applications of these results in insurance and finance up to 2002 can be found in Dhaene et al. (2002b).

\subsection{Sums of comonotonic random variables}

Consider a random vector $\left(X_{1}, \ldots, X_{n}\right)$ and its comonotonic counterpart $\left(X_{1}^{c}, \ldots, X_{n}^{c}\right)$. The sum of the components of $\left(X_{1}^{c}, \ldots, X_{n}^{c}\right)$ is denoted by $S^{c}$,

$$
S^{c}=X_{1}^{c}+\cdots+X_{n}^{c} .
$$

The distribution of the comonotonic sum $S^{c}$ can be determined from

$$
F_{S^{c}}(x)=\sup \left\{p \in[0,1] \mid \sum_{i=1}^{n} F_{X_{i}}^{-1}(p) \leq x\right\}, \quad x \in \mathbb{R} .
$$

The distribution of $S^{c}$ can also be specified via its quantile function $F_{S^{c}}^{-1}(p)$, which exhibits the following additivity property:

$$
F_{S^{c}}^{-1}(p)=\sum_{i=1}^{n} F_{X_{i}}^{-1}(p), \quad p \in[0,1] .
$$


Hereafter, we will always assume that all random variables $X_{i}$ have finite means. The distribution of $S^{c}$ can then be specified via its stop-loss transform E $\left[\left(S^{c}-x\right)_{+}\right]$. Dhaene et al. (2000) show that any stop-loss premium $\mathrm{E}\left[\left(S^{c}-x\right)_{+}\right]$can be decomposed into a linear combination of stop-loss premiums $\mathrm{E}\left[\left(X_{i}-x_{i}\right)_{+}\right], i=1,2, \ldots, n$, for appropriate choices of the $x_{i}$.

In order to state this decomposition formula more formally, we first introduce other types of generalized inverses of cdf's. The càdlàg inverse $F_{X_{i}}^{-1+}$ is defined by:

$$
F_{X_{i}}^{-1+}(p)=\sup \left\{x \in \mathbb{R} \mid F_{X_{i}}(x) \leq p\right\}, \quad p \in[0,1],
$$

with $\sup \emptyset=-\infty$, by convention. Following Kaas et al. (2000), for any $\alpha \in[0,1]$, the inverse $F_{X_{i}}^{-1(\alpha)}$ is defined by

$$
F_{X_{i}}^{-1(\alpha)}(p)=\alpha F_{X_{i}}^{-1}(p)+(1-\alpha) F_{X_{i}}^{-1+}(p), \quad p \in(0,1) .
$$

The decomposition formula of Dhaene et al. (2000) can then be expressed as follows:

$$
\mathrm{E}\left[\left(S^{c}-x\right)_{+}\right]=\sum_{i=1}^{n} \mathrm{E}\left[\left(X_{i}-F_{X_{i}}^{-1(\alpha)}\left(F_{S^{c}}(x)\right)_{+}\right], \quad x \in\left(F_{S^{c}}^{-1+}(0), F_{S^{c}}^{-1}(1)\right) .\right.
$$

Here, $\alpha$ is any element of $[0,1]$ satisfying

$$
\sum_{i=1}^{n} F_{X_{i}}^{-1(\alpha)}\left(F_{S^{c}}(x)\right)=x
$$

A special case of the decomposition formula (5) can be found in Jamshidian (1989) who proves that in the Vasicek (1977) model, a European option on a portfolio of pure discount bonds (in particular, an option on a coupon-bearing bond) decomposes into a portfolio of European options on the individual discount bonds in the portfolio. This holds true because in the Vasicek model, the prices of all pure discount bonds at some future time $T$ are decreasing functions of a single random variable, namely the spot rate at that time. This implies that the price at time $T$ of the portfolio of pure discount bonds is a comonotonic sum. Taking into account that the current price of a European option can be expressed as the discounted expected pay-off of this option, where the expectation is taken with respect to an appropriate measure, we find that for the current price of the option on the portfolio of zero coupon bonds a decomposition as in (5) holds.

\subsection{Convex bounds for sums of random variables}

Consider a random vector $\underline{X} \equiv\left(X_{1}, X_{2}, \ldots, X_{n}\right)$, not necessarily comonotonic, and the sum of its components

$$
S=X_{1}+\cdots+X_{n}
$$

Intuitively, one might expect that the comonotonic sum $S^{c}=X_{1}^{c}+\cdots+X_{n}^{c}$ of the comonotonic counterpart $\underline{X}^{c}$ is more variable than the original sum $S$. In order to state this intuitive result more formally, we need the notion of convex order.

A random variable $X$ is said to precede a random variable $Y$ in the convex order sense, notation $X \leq_{\mathrm{cx}} Y$, if the following conditions hold:

$$
\mathrm{E}\left[(x-X)_{+}\right] \leq \mathrm{E}\left[(x-Y)_{+}\right], \text {for all } x,
$$

and

$$
\mathrm{E}\left[(X-x)_{+}\right] \leq \mathrm{E}\left[(Y-x)_{+}\right], \text {for all } x \text {. }
$$


Other characterizations of convex order can be found, e.g., in Shaked and Shanthikumar (1994) in a general context, or in Denuit et al. (2005) in an actuarial context. Intuitively, the convex order relation $X \leq_{\mathrm{cx}} Y$ states that compared to the random variable $X$, the random variable $Y$ has more probability mass in its lower as well as its upper tails. Wang and Young (1998) compare the concept of ordering random variables in expected utility theory versus Yaari's dual theory of choice under risk.

One can prove that the following relation holds between the sum $S$ and its comonotonic modification $S^{c}$ :

$$
X_{1}+\cdots+X_{n} \leq_{\mathrm{cx}} X_{1}^{c}+\cdots+X_{n}^{c}=S^{c} .
$$

This result states that when one assumes that only the marginal distributions of the random variables are given (or used), the largest sum in convex order occurs when the random variables are comonotonic. To the best of our knowledge, this result was first mentioned in the actuarial literature in Heilmann (1986), who attributes it to Meilijson and Nadas (1979). Other early references are Tchen (1980) and Rüschendorf (1983). Tchen (1980) has proven that in the class of all random vectors with given marginals the comonotonic random vectors are greater in supermodular order than any other element of this class. A simple proof for the inequality (6), which is based on a geometric interpretation of the support of the comonotonic distribution, is given in Kaas et al. (2002).

Since the mid 1990's the convex order relation (6) has attracted a lot of attention in the actuarial literature. Dhaene and Goovaerts (1996, 1997), Müller (1997), Bäuerle and Müller (1998), Wang and Dhaene (1998), Goovaerts and Dhaene (1999) and Denuit et al. (2001) generalize (6) by investigating how changing the dependence structure of an insurance portfolio influences its stop-loss premiums. In any of the different situations considered in these papers, the convex order relation (6) corresponds with the extreme case where the comonotonic dependence structure is involved.

From the convex order relation (6), it follows immediately that $\mathrm{E}\left[\left(S^{c}-x\right)_{+}\right]$can be interpreted as the solution to the following maximization problem, where we use $R_{n}$ as a shorthand notation for $R_{n}\left(F_{X_{1}}, F_{X_{2}}, \ldots, F_{X_{n}}\right)$ :

$$
\max _{\underline{Y} \in R_{n}} \mathrm{E}\left[\left(Y_{1}+Y_{2}+\ldots+Y_{n}-x\right)_{+}\right]=\mathrm{E}\left[\left(X_{1}^{c}+\cdots+X_{n}^{c}-x\right)_{+}\right], \quad x \in \mathbb{R} .
$$

This means that $\mathrm{E}\left[\left(S^{c}-x\right)_{+}\right]$can be interpreted as an extreme-case expectation for $\mathrm{E}\left[(S-x)_{+}\right]$. Indeed, let us assume that the only information that is available about the distribution of the random vector $\underline{X}$ are the marginal cdf's $F_{X_{i}} i=1, \ldots, n$. In this case, the largest possible value for $\mathrm{E}\left[(S-x)_{+}\right]$is given by $\mathrm{E}\left[\left(S^{c}-x\right)_{+}\right]$.

One can also prove that $\mathrm{E}\left[\left(S^{c}-x\right)_{+}\right]$is the solution to the following minimization problem:

$$
\min _{\sum_{i=1}^{n} x_{i}=x} \sum_{i=1}^{n} \mathrm{E}\left[\left(X_{i}-x_{i}\right)_{+}\right]=\mathrm{E}\left[\left(X_{1}^{c}+\cdots+X_{n}^{c}-x\right)_{+}\right],
$$

where the minimum is taken over all $\left(x_{1}, x_{2}, \ldots, x_{n}\right)$ with $\sum_{i=1}^{n} x_{i}=x$, see Rüschendorf (1983).

To the best of our knowledge, Simon et al. (2000) were the first who combined the convex order relation (6) and the decomposition formula (5) to find an upper bound for the price of an arithmetic European type Asian option in terms of the price of an appropriate portfolio of plain vanilla European call options. Furthermore, from the optimization result (7), they conclude that the exercise prices of the plain vanilla options contained in their upper bound is optimal in the sense that no improvement can be obtained by considering other linear combinations of plain vanilla European options. Important to notice is that this result is model-independent. Later, Albrecher et al. (2005) interpret the comonotonic upper bound of Simon et al. (2000) as the 
price of a static superhedging strategy for an Asian option, where the hedging portfolio consists of plain vanilla options. Using static superhedging strategies has the advantage that it is much less sensitive to the assumption of zero transaction costs and to the hedging performance in the presence of large market movements, compared to dynamic strategies.

In order to be able to determine the upper bound in (6), the only information that is required about the distribution of $\underline{X}$ is its marginals. Intuitively, it is clear that it must be possible to find better convex order upper bounds for $S$ when more information is available concerning the multivariate cdf of $\underline{X}$. Therefore, let us assume that apart from the knowledge of the marginals, there exists a random variable $\Lambda$ with a given distribution function, such that the conditional distributions of the random variables $X_{i}$, given $\Lambda=\lambda$, are known for all outcomes $\lambda$ of $\Lambda$. Kaas et al. (2000) derive the following improved convex order upper bound, denoted $S^{i c}$, for this particular case:

$$
X_{1}+\cdots+X_{n} \leq_{\mathrm{cx}} F_{X_{1} \mid \Lambda}^{-1}(U)+F_{X_{2} \mid \Lambda}^{-1}(U)+\ldots+F_{X_{n} \mid \Lambda}^{-1}(U)=S^{i c},
$$

where $F_{X_{i} \mid \Lambda}^{-1}(U)$ is a notation for the random variable $f_{i}(U, \Lambda)$, with $f_{i}$ defined by $f_{i}(u, \lambda)=$ $F_{X_{i} \mid \Lambda=\lambda}^{-1}(u)$. Notice that the random vector $\left(F_{X_{1} \mid \Lambda}^{-1}(U), F_{X_{2} \mid \Lambda}^{-1}(U), \ldots, F_{X_{n} \mid \Lambda}^{-1}(U)\right)$ is said to be 'conditionally comonotonic'.

Based on an idea that stems from mathematical physics, Kaas et al. (2000) propose the following convex order lower bound for $S$, denoted $S^{\ell}$, when the information available about the cdf of $\underline{X}$ is the same as the one that leads to the upper bound in (8):

$$
S^{\ell}=\mathrm{E}\left[X_{1} \mid \Lambda\right]+\mathrm{E}\left[X_{2} \mid \Lambda\right]+\cdots+\mathrm{E}\left[X_{n} \mid \Lambda\right] \leq_{c x} X_{1}+X_{2}+\cdots+X_{n} .
$$

They remark that this lower bound has the nice property that it is a comonotonic sum, provided all terms $\mathrm{E}\left[X_{i} \mid \Lambda\right]$ are increasing (or all are decreasing) functions of $\Lambda$. In this case, the quantiles and stop-loss premiums of $S^{\ell}=\sum_{i=1}^{n} \mathrm{E}\left[X_{i} \mid \Lambda\right]$ follow immediately from the additivity properties of comonotonic sums in (2.1) and (5). This property is particularly of interest in a multivariate lognormal setting. In such a setting, the lower bounds turn out to be very accurate, provided the appropriate choice is made for the conditioning random variable $\Lambda$, see, e.g., Vanduffel et al. (2005b).

The lower bound (9) is applied in Dhaene et al. (2002b) to derive accurate approximations for European type Asian options in a Black \& Scholes setting, in case of discrete averaging of the stock price. In a lognormal setting, Rogers and Shi (1995) apply a similar lower bound to derive approximations for the price of Asian options in case of continuous averaging.

\section{$3 \quad$ Further developments of the theory}

In this section we summarize several extensions of the theory of comonotonicity since 2004, not claiming to be exhaustive but trying to be as complete as possible, taking into account that this theory is still in development.

The inequality (6) implies that if a random vector with given marginal distributions is comonotonic, it has the largest sum with respect to convex order. Cheung (2008b) proves that the converse holds also true, provided that each marginal distribution is continuous.

Defining the improved comonotonic upper bound, see relation (8), Kaas et al. (2000) introduced implicitly the notion of conditional comonotonicity. This notion is later more formally considered by Jouini and Napp (2004) as a generalization of the classical concept of comonotonicity. In Cheung (2007b), this concept is further investigated. The main result is that 
a random vector is comonotonic conditional to a certain sigma-field if and only if it is almost surely comonotonic locally on each atom of the conditioning sigma-field. In Cheung (2008a), the relationship between conditional comonotonicity and convex ordering is explored. By this notion of conditional comonotonicity it is possible to unify the classical upper bound result (inequality (6)) and the improved upper bound result (inequality (8)) in a more general framework.

The choice of the conditioning random variable $\Lambda$ in (9) is crucial for the accuracy of the lower bound approximation $S^{\ell}$. When $S$ is a sum of non-independent lognormal random variables, different alternatives for $\Lambda$ have been proposed in the literature, see e.g. Kaas et al. (2000) and Vanduffel et al. (2005b). These choices were 'global' in the sense that $\Lambda$ was chosen such that the entire distribution of the approximation $\mathrm{E}[S \mid \Lambda]$ is 'close' to the corresponding distribution of the original sum $S$. In an actuarial or a financial context one is often only interested in a particular tail of the distribution of $S$. Therefore Vanduffel et al. (2008a) propose locally optimal approximations, in the sense that the relevant tail of the distribution of $\mathrm{E}[S \mid \Lambda]$ is an accurate approximation for the corresponding tail of the distribution of $S$. Deelstra et al. (2009a) study sums $S$ of the form $\sum_{i=1}^{n} w_{i} \alpha_{i} e^{\beta_{i}+\gamma_{i} Y_{i}}$ where the positive weights $w_{i}$ sum up to one, the coefficients $\alpha_{i}(>0), \beta_{i}, \gamma_{i}$ are deterministic and the $Y_{i}$ 's are non-independent normally distributed random variables. In this case, Deelstra et al. (2009a) show that all these choices for the conditioning random variable $\Lambda$ can be considered as a linear transformation of a first order approximation of $S$, namely $\Lambda=\sum_{i=1}^{N} w_{i} \alpha_{i} \gamma_{i} Y_{i} \delta_{i}$ with $\delta_{i}$ taking different forms according to the different choices.

The applicability of the convex bounds (6), (8) and (9) to derive closed-form approximations for risk measures of a sum of non-independent lognormal random variables with unknown dependence structure is illustrated in Dhaene et al. (2002b). Valdez et al. (2009) investigate to which extent the general results on convex bounds of Section 2 can be applied to sums of non-independent log-elliptical random variables which incorporate sums of log-normals as a special case. First, they show that unlike the log-normal case, for general sums of log-ellipticals the convex lower bound (9) does no longer result in closed-form approximations for the different risk measures. Second, they demonstrate how instead the weaker stop-loss order can be used to derive such closed-form approximations. In numerical illustrations they show that these newly proposed approximations are useful to measure satisfactorily the risk of discounted or compounded sums in case the stochastic returns are elliptically distributed.

More general, Kukush and Pupashenko (2007) study comonotonic upper and lower bounds for sums under a mixture of arbitrary distributions. They also consider the case where the logarithm of the components in the sum can be represented as a mixture of normal random variables. These results may be useful to perform approximate evaluations of actuarial provisions when a regime switching model is used for the investment returns.

Yang et al. (2006) investigate bivariate copula structures for modeling dependence among variables in a distribution free way. The existence and uniqueness of a bivariate copula decomposition into a comonotonic, an independent, a countermonotonic and an indecomposable part are proved, while the coefficients are determined from partial derivatives of the corresponding copula. Moreover, for the indecomposable part, an optimal convex approximation is provided and analyzed. The variance decomposition that they derive, can be applied to find mean-variance optimal investment portfolios in finance. They also give other applications of this decomposition in finance and insurance.

Hoedemakers et al. (2005) and Ahcan et al. (2006) extend the theory of convex bounds to the case of scalar products of mutually independent random vectors. This methodology allows one to obtain reliable approximations of the underlying distribution functions as well as very accurate estimates of upper quantiles and stop-loss premiums. Hua and Cheung (2008a) also study stochastic orders of scalar products of random vectors and derive more general conditions 
under which linear combinations of random variables can be ordered in the increasing convex order.

Cheung (2009) introduces upper comonotonicity as a generalization of the classical notion of comonotonicity. A random vector $\underline{X}=\left(X_{1}, X_{2}, \ldots, X_{n}\right)$ is said to be upper-comonotonic if its components $X_{i}$ are moving in the same direction simultaneously when their values are greater than some thresholds.

This new notion can be characterized in terms of both the joint distribution function and the underlying copula. The copula characterization allows the study of the coefficient of upper tail dependence as well as the distributional representation of an upper-comonotonic random vector. The additivity property of several commonly used risk measures, such as the Value-at-Risk, the Tail Value-at-Risk and the expected shortfall for sums of comonotonic risks is extended to sums of upper-comonotonic risks, provided that the level of probability is greater than a certain threshold.

For premium calculation principles or risk measures, usually only the additivity for a finite number of comonotonic risks is considered. However, a limiting status of finite additivity is the additivity for countable risks. In Wu and Zhou (2006), the countable additivity is investigated and new and elegant characterizations for Choquet pricing and distortion premium principles are presented. The countable exchangeability is also studied following the investigation of countable additivity for comonotonic risks. It leads to generalized Choquet pricing and generalized distortion premium principles.

Several multivariate extensions of comonotonicity are studied in Puccetti and Scarsini (2010). Naive extensions do not enjoy some of the main properties of the univariate concept. In the univariate case, the definition of comonotonicity only relies on the total order structure. Hence this definition could be extended for any random vector with values in a product of totally ordered measurable spaces. Most of its properties would be valid even in this multivariate context. However, the aim of Puccetti and Scarsini is to study comonotonic vectors that take values in a product of partially ordered spaces. Different definitions of multivariate comonotonicity are introduced, trying to extend different features of the classical definition. It is shown that no definition satisfies all the properties of the original one. Some definitions do not guarantee the existence of a comonotonic random vector for any pair of multivariate marginals. Some other definitions do not guarantee uniqueness in distribution of the comonotonic random vector with fixed marginals.

In finance, Galichon and Henry (2008) and Ekeland et al. (2009) propose a multivariate extension of coherent risk measures that involves a multivariate extension of the notion of comonotonicity, in the spirit of Puccetti and Scarsini (2010).

\section{Applications of the theory of comonotonicity}

\subsection{Derivatives pricing and hedging}

Several European options have a pay-off written on one or multiple underlyings combined in a weighted sum of non-independent random variables expressing asset prices at the time of maturity or at different time points before and at maturity. Examples of this type of options with positive weights are Asian options, basket options and Asian basket options. When the weights can be both positive and negative, one refers to these options as spread options, Asian spread options, basket spread options and Asian basket spread options. Pricing and hedging of these products by means of comonotonicity bounds has been studied in a model dependent as well as in a model independent framework. As mentioned before, early references to this topic 
are Rogers and Shi (1995), Simon et al. (2000) and Dhaene et al. (2002b). Hereafter, we will discuss articles published since 2004 dealing with this topic.

First we consider the model dependent setting. A survey of current methods up to 2006 for pricing Asian options and computing their sensitivities to the key input parameters is provided in Boyle and Potapchik (2008). The methods discussed there include also the comonotonic bounds. We will focus in our present paper only on those papers dealing with comonotonic bounds. In comparison with Boyle and Potapchik (2008), we will also discuss more recent papers and other applications.

Schrager and Pelsser (2004) use a change of numeraire technique to derive a general pricing formula for the Rate of Return Guarantees in a Regular Premium Unit Linked (UL) Insurance contract. They show that the guarantee is equivalent to a European put option on some stochastically weighted average of the stock price at maturity. They extend earlier results from Simon et al. (2000) and Dhaene et al. (2002b) on pricing bounds of Asian options to UL Guarantees and stochastic interest rates in the case that the underlying sum is composed of lognormal random variables.

In Vanmaele et al. (2006), the pricing of European-style discrete arithmetic Asian options with fixed and floating strike is studied by deriving analytical lower and upper bounds, as explained in Section 2, and additionally combined with the ideas of Simon et al. (2000) and of Nielsen and Sandmann (2003). Through these bounds, a unifying framework is created for European-style discrete arithmetic Asian options, that generalizes several approaches in the literature as well as improves existing results. Analytical and easily computable bounds are obtained under the Black and Scholes model for the asset prices. An advice of the appropriate choice of the bounds given the parameters is formulated, the effect of different conditioning variables is investigated and their efficiency is numerically compared. Based on these approximating bounds analytical hedging formulas are developed.

Making use of geometric arguments, Brückner (2008) quantifies the maximal error in terms of truncated first moments, when a sum $S$ is approximated by the upper bound $S^{c}$ or a lower bound $S^{\ell}$ as defined in (6) and (9), respectively.

Vyncke et al. (2004) construct a convex combination of the comonotonic upper bound and the lower bound for the price of a European-style arithmetic Asian option and find an approximation for this price which is such that the underlying approximate cdf has exact first and second moments.

Inspired by the ideas of Rogers and Shi (1995), Chalasani and Varikooty (1998) derived accurate lower and upper bounds for the price of a European-style Asian option with continuous averaging over the full lifetime of the option, using a discrete-time binary tree model. Reynaerts et al. (2006) consider arithmetic Asian options with discrete sampling and they generalize the method of Chalasani and Varikooty (1998) to the case of forward starting Asian options. In this case with daily time steps, that method is still very accurate but the computation can take a very long time on a PC when the number of steps in the binomial tree is high. Reynaerts et al. (2006) derive analytical lower and upper bounds based on the results presented in Section 2, and by conditioning on the value of the underlying asset at the exercise date. The comonotonic upper bound corresponds to an optimal superhedging strategy. By putting in less information than Chalasani and Varikooty (1998) the bounds lose some accuracy but are still very good and they are easily computable and moreover the computation on a PC is fast.

Also the price of a continuously sampled European-style Asian option with fixed exercise price can be approximated by means of the tools of Section 2. Within a Black and Scholes framework, Vanduffel et al. (2008b) derive analytic expressions for lower and upper bounds for such a price.

As for Asian options, determining the price of a European basket option is not a trivial task, 
even in the Black and Scholes model, because there is no explicit analytical expression available for the distribution of the weighted sum of prices of the assets in the basket. The upper bounds proposed in Section 2 will not always lead to good approximations since a basket of underlyings can be far from a comonotonic sum, depending on the correlations between the assets. However, by using a conditioning variable, the price of a European basket option can be decomposed in two parts, one of which can be computed exactly. For the remaining part Deelstra et al. (2004) derive a lower and some upper bounds based on the theory of comonotonicity. The lower bound obtained in this way corresponds to (5) with $S^{\ell}$ introduced in (9) being the comonotonic sum $S^{c}$. The first upper bound is based on an improved comonotonic upper bound upon the part in the pricing formula that cannot be calculated in an explicit way. The other upper bound is obtained by using the ideas of Rogers and Shi (1995) and Nielsen and Sandmann (2003) upon that same part. By concentrating only upon this inexact part, much preciser approximating bounds can be obtained.

The lower bounds and some of the upper bounds discussed above are based on comonotonicity results combined with conditioning upon one variable. In a Black and Scholes setting, Vanmaele et al. (2004) derive analytical expressions for comonotonic bounds of stop-loss premiums of sums of non-independent random variables by conditioning upon two variables. They also use the idea of several conditioning variables to develop an approximation for cases for which it is cumbersome to obtain a comonotonic lower bound. The numerical application to European basket options shows that conditioning on two variables leads to very sharp results.

Combining the features of Asian and basket options we end up with European-style discrete arithmetic Asian basket options. Deelstra et al. (2008) propose pricing bounds for these options in a Black and Scholes framework. They use the general approach for deriving upper and lower bounds as in Section 2 and generalize in this way the methods of Deelstra et al. (2004) and Vanmaele et al. (2006). They further show how to derive an analytical closed-form expression for a lower bound in the non-comonotonic case. Finally, in numerical tests the quality of these bounds are compared to upper bounds for Asian basket options based on techniques as in Thompson (1999) and Lord (2006).

When allowing also for negative weights one can price European-style discrete arithmetic Asian basket spread options. Deelstra et al. (2009b) derive comonotonic lower and upper bounds for such spread options and discuss the behaviour of these approximating bounds. They also develop a new hybrid moment matching method, namely a moment matching of both the positively weighted basket and the negatively weighted basket separately, combined with an improved comonotonic upper bound (5) with $S^{i c}$ from (8) being the comonotonic sum $S^{c}$. Deelstra et al. (2009b) find that the improved comonotonic upper bound offers a good approximation of the price of spread options. The hybrid moment matching method based upon the improved comonotonic upper bound approach leads to a well-performing bound for Asian basket spread options. The Greeks for these two methods are explicitly derived. Moreover, the results can be extended to options denominated in foreign currency.

Deelstra et al. (2010) elaborate a method for determining the optimal strike price for a put option, used to hedge a position in a financial product such as a basket of shares or a couponbearing bond. This strike price is optimal in the sense that it minimizes, for a given budget, a class of risk measures satisfying certain properties. Hereto they study the loss function in the worst case scenario such that its risk is on the safe side. Formulas are derived for one single underlying as well as for a weighted sum of underlyings. For the latter, two cases are considered depending on the dependence structure of the components in this weighted sum, namely the case that the components form a comonotonic vector and the case that they are not comonotonic. In the latter case comonotonic approximations based on $S^{c}(4)$, respectively on $S^{\ell}(9)$, are proposed. 
Now we turn to the model independent bounds as the ones presented in Simon et al. (2000) and Albrecher et al. (2005) for Asian options.

In Hobson et al. (2005a,b) static-arbitrage super-replicating respectively subreplicating strategies for European-style basket options are derived. In the former article, the authors consider the set of all models which are consistent with the observed prices of vanilla options, and, within this class, find the model for which the price of the basket option is largest. This price is an upper bound on the prices of the basket option which are consistent with no-arbitrage. In the absence of additional assumptions it is the lowest upper bound on the price of the basket option and is related to a comonotonic upper bound. Both the infinite market case (where prices of the plain vanilla options are available for all strikes) and the finite market case (where only a finite number of plain vanilla option prices are observed) are considered. From a pure mathematical point of view, the infinite market case results are closely related to the optimization result (7) presented in Section 2. In the latter article, subreplicating strategies are developed for European-style basket options consisting of two assets. The so-called sheeptrack portfolio has a price that can only be realized by a countermonotonic pair as defined in (3).

Whereas Hobson et al. (2005a) only concentrate on basket options, Chen et al. (2008) investigate static super-replicating strategies for European-type call options written on a weighted sum of asset prices. This class of exotic options includes Asian options and basket options among others. It is assumed that there exists a market where the plain vanilla options on the different assets are traded and hence their prices can be observed in the market. Both the infinite and the finite market case are considered. It is proven that the finite market case converges to the infinite market case when the number of observed plain vanilla option prices tends to infinity. The paper shows how to construct a portfolio consisting of the plain vanilla options on the different assets, whose pay-off super-replicates the pay-off of the exotic option. As a consequence, the price of the super-replicating portfolio is an upper bound for the price of the exotic option. The superhedging strategy is model-free in the sense that it is expressed in terms of the observed option prices on the individual assets, which can be e.g. dividend paying stocks with no explicit dividend process known. As opposed to Hobson et al. (2005a) who use Lagrange optimization techniques, the proofs in Chen et al. (2008) are based on the theory of integral stochastic orders, comonotonicity and convex bounds, see Sections 1 and 2 .

Chen et al. (2009) further investigate super-replicating strategies for European-type call options written on a positively weighted sum of asset prices following the initial approach in Chen et al. (2008). To be more precise, three issues are proposed and investigated concerning the optimal super-replicating strategies. The first issue is the non-uniqueness of the optimal solution. The second issue is to generalize the results from a deterministic interest rate setting in the previous paper to a stochastic interest rate setting. By performing this generalization, optimal super-replicating strategies are obtained in a more general market. The third issue is about the co-existence of the comonotonicity property and the martingale property. When there is only one underlying asset, it is shown that they possibly co-exist for some cases, while for some other cases there can also be a contradiction between them. As a consequence, for Asian options, the upper bound may not be reachable in an arbitrage-free market.

Distribution-free bounds in closed-form and optimal hedging strategies for spread options are derived in Laurence and Wang $(2008,2009)$. The former article focuses on upper bounds when the spread option's joint distribution is calibrated to the information about the marginals embedded in the prices of traded options with all available strikes of a given maturity.

In the latter article, sharp distribution-free lower bounds for spread options and the corresponding optimal subreplicating portfolios are obtained. This lower bound is attained for the comonotonic distributions. Laurence and Wang also introduce the notion of monotonicity gap which can be further divided into two complementary gaps: the countermonotonicity and the comonotonicity gap. The idea is that the normalized distance of the true (quoted) market price 
of a spread option from the distribution free comonotonic upper bound (respectively, countermonotonic lower bound) represents a useful and new 'market implied' index. This index measures how far the assets are from being countermonotonic (respectively, comonotonic) and can be used as a distribution free complement to the so-called implied correlation that is widely used in the industry.

Finally, we draw the attention to some recent articles where comonotonicity is applied to price or hedge some other types of financial products.

Based on the positive dependence characteristic of the mortality in catastrophe areas, Shang et al. (2009) develop a pricing model for catastrophe mortality bonds with comonotonicity and a jump-diffusion process. Since there is no unique risk-neutral probability in this incomplete market settings, they use the Wang transform method to price the bond.

In Burtschell et al. (2009) possible bounds on CDO tranche premiums are studied. In case of a comonotonic vector of default times a model-free lower bound on equity tranche premiums is provided, where model-free has to be understood with respect to the dependence structure between default dates. The CDO tranche premiums computations turn out to be straightforward in this comonotonic case.

Glau et al. (2009) study interest rate derivatives. In particular, they consider the Lévy term structure model that extends the Heath-Jarrow-Morton model in that the instantaneous forward rate is given by a time-inhomogeneous Lévy process. Within this framework pricing formulas based on Fourier transforms are known for the most liquid interest rate derivatives, namely caps, floors and swaptions. Glau et al. (2009) study delta-hedging and risk-minimizing hedging strategies for swaptions on the basis of zero coupon bonds. They derive closed-form expressions for the hedging strategy in terms of the Fourier transforms by the comonotonicity property.

\subsection{Risk management: risk sharing, optimal investment, capital allocation}

\subsubsection{Risk measures and risk sharing}

In Dhaene et al. (2002b) it is shown how the convex bounds (6), (8) and (9) can be used to derive closed-form approximations for risk measures of a sum of non-independent lognormal random variables. Dhaene et al. (2006b) further examine and summarize properties of several well-known risk measures that can be used in the framework of setting capital requirements for a risky business. Special attention is given to the class of concave distortion risk measures also called spectral distortion risk measures, see Acerbi (2002). Note that the class of concave distortion risk measures is a subset of the more general class of coherent risk measure as introduced in Artzner (1999) and Artzner et al. (1999), see also Huber (1981).

Dhaene et al. (2006b) investigate the relationship between these risk measures and theories of choice under risk. They further consider the problem of how to evaluate these risk measures for sums of non-independent random variables and approximations for such sums, based on the concept of comonotonicity, are proposed. Another generalization of the class of concave distortion risk measures in a distribution free setting is considered in Dhaene et al. (2006a).

Goovaerts et al. (2004) present a new axiomatic characterization of risk measures that are additive for independent random variables. The axiom of additivity for independent random variables is related to an axiom of additivity for comonotonic random variables. The risk measures characterized can be regarded as mixed exponential premiums.

The appropriateness of the subadditivity of risk measures is considered in Dhaene et al. (2008b).

Dhaene et al. (2009a) investigate the influence of the dependence between random losses on the shortfall and on the diversification benefit that arises from merging these losses. They prove that increasing the dependence between losses, expressed in terms of correlation order, has an 
increasing effect on the shortfall, expressed in terms of an appropriate integral stochastic order. Furthermore, increasing the dependence between losses decreases the diversification benefit. In particular, they consider merging comonotonic losses and show that even in this extreme case a non-negative diversification benefit may arise.

Embrechts et al. (2005) prove that comonotonicity gives rise to the on-average-most-adverse Value-at-Risk (VaR) scenario for a function of dependent risks, when the marginal distributions are known but the dependence structure between the risks is unknown. Laeven (2009) extends this result to the case where, rather than no information, partial information is available on the dependence structure between the risks. Moreover, Laeven (2009) points out that the improved comonotonic or conditionally comonotonic dependence structure as introduced in (8) is very interesting as a worst-case scenario. Indeed, it is the most adverse dependence structure in stop-loss and supermodular order and hence in Tail-VaR-based risk management, and the onaverage-most-adverse dependence structure in VaR-based risk management.

Tsanakas and Christofides (2006) model an exchange economy where agents (insurers/banks) trade risks. Decision making takes place under distorted probabilities, which are used to represent either rank-dependence of preferences or ambiguity with respect to real-world probabilities. Via the construction of aggregate preferences from heterogeneous agents' utility and distortion functions, they obtain pricing formulas and risk allocations, generalizing results of Bühlmann (1970). In particular, in a lemma which can be viewed as a generalized version of Borch's characterization of Pareto optima, it is stated that at equilibrium the agents' risk allocations are comonotonic random variables.

Jouini et al. (2008) consider the problem of optimal risk sharing of some given total risk between two economic agents characterized by law-invariant monetary utility functions or equivalently, law-invariant risk measures. In the case that both agents' utility functions are comonotone an explicit characterization of an optimal risk sharing allocation is provided. This optimal allocation is in addition increasing in terms of the total risk.

Also in Ludkovski and Rüschendorf (2008) the risk sharing problem is dealt with. They extend the result that a Pareto optimal risk allocation is necessarily comonotone to the case of unbounded random variables and this for certain classes of consistent risk measures. This is significant from a practical point of view where risks are often modeled as unbounded random variables.

\subsubsection{Optimal investment strategies}

Dhaene et al. (2005) investigate multiperiod portfolio selection problems in a Black and Scholes type market where a basket of one riskfree and $m$ risky securities are traded continuously. They look for the optimal allocation of wealth within the class of constant-mix portfolios. First, they consider the portfolio selection problem of a decision maker who invests money at predetermined points in time in order to obtain a target capital at the end of the time period under consideration. A second problem concerns a decision maker who invests some amount of money (the initial wealth or provision) in order to be able to fulfil a series of future consumptions or payment obligations. Several optimality criteria and their interpretation within Yaari's dual theory of choice under risk are presented. For both selection problems, accurate approximations are proposed based on the concept of comonotonicity as exposed in Section 2. Similar problems are considered in the related papers Vanduffel et al. (2003, 2005a).

Cheung (2006) and Cheung and Yang (2008) study a single-period optimal portfolio problem. It is assumed that the actual dependence structure of the asset returns is unknown or is a mixture of some common underlying source of risks. The least favourable dependence structure is first identified, then the optimal portfolio problem is analyzed as if this were the actual dependence 
structure. A sufficient condition to order the optimal allocations is obtained using concepts of stochastic ordering.

\subsubsection{Capital allocation}

The Enterprise Risk Management process of a financial institution usually contains a procedure to allocate, or subdivide, the total risk capital of the company into its different business units.

In Dhaene et al. (2003), an optimization argument is used to find an optimal rule for allocating the aggregate capital of a financial firm to its business units. The optimal allocation can be found using general results from the theory on comonotonicity as summarized in Section 2 . Dhaene et al. (2009b) generalize the approach of Dhaene et al. (2003) and develop a unifying framework for allocating the aggregate capital by considering more general deviation measures. Capital allocation based on the principle of comonotonicity turns out to be a special case of this general framework, as well as many other allocation rules that are described in the literature.

Taking the viewpoint of a higher authority within the financial conglomerate (typically the board of directors) by which the economic capital allocation is performed, Laeven and Goovaerts (2004) propose an optimization approach to allocate economic capital, distinguishing between an allocation or raising principle, and a measure for the risk residual. The approach provides an integrated solution since it can be applied both at the aggregate (conglomerate) level and at the individual (subsidiary) level. Different degrees of information on the dependence structure between the subsidiaries are considered. When using expectations as risk measure and assuming a complete lack of information on the dependence structure between the subsidiaries, the capital allocation problem reduces to the problem considered in Dhaene et al. (2003).

Dhaene et al. (2008a) study the CTE-based allocation rule, where the Conditional Tail Expectation (CTE) acts as risk measure to deal with the allocation problem. Comonotonicity is used to derive accurate and easy to compute closed-form approximations for the CTE-based allocation rule. Hence, the field where analytical solutions for this rule are available is extended to the case that the risks of the different units have a multivariate (log)normal distribution.

Cheung (2007a) studies orderings of optimal allocations of policy limits and deductibles when losses are cumulative, while Hua and Cheung (2008a) introduce new models to separate the effects of severities and frequencies of losses. In both of these papers the study is carried out from the viewpoint of a risk-averse policyholder and under the assumption that the dependence structure of the losses is unknown. In order to deal with this, they focus on the worst allocation problem, the worst dependence structure being identified as the comonotonic one. In Hua and Cheung (2008b) the worst allocations of policy limits and deductibles is studied from the viewpoint of an insurer. The main results of these articles are complemented and extended in Zhuang et al. (2009) by applying bivariate characterizations of stochastic ordering relations.

Tsanakas (2008) constructs a distortion-type risk measure, which evaluates the risk of any uncertain position in the context of a portfolio that contains that position and a fixed background risk, which means that besides the specific portfolio, the holder is also exposed to a risk that he cannot (or will not) trade, control or mitigate. The risk measure can also be used to assess the performance of individual risks within a portfolio, allowing for the portfolio's re-balancing, an area where standard capital allocation methods fail. It is shown that the properties of the risk measure depart from those of coherent distortion measures. In particular, it is shown that the presence of background risk makes risk measurement sensitive to the scale and aggregation of risk. However, the risk of an instrument $X$ relative to a background risk $Y$, which is comonotone to $X$, is equal to the risk of $X$ with no background risk. Further, the case of risks following elliptical distributions is examined in more detail and precise characterizations of the risk measure's aggregation properties are obtained. 
Tsanakas (2009) discusses the use of convex risk measures in capital allocation. He studies a flexible class of convex risk measures, namely the distortion-exponential risk measure depending on a positive real number and a concave, differentiable distortion function. For extreme cases of dependence between the risks, such as comonotonicity or countermonotonicity, see Section 1 , the aggregation properties of this convex risk measure are characterized and explicit capital allocation formulas are obtained.

\subsection{Life Insurance and pensions}

In the classical approach to the theory of life contingencies, discounting factors and mortality tables are assumed to be deterministic. In view of the long durations of life annuity contracts it is more realistic to take the stochastic nature of investment returns and mortality into account when investigating the risks related to annuity portfolios. Over the last two decades, a large number of papers have been published covering this stochastic approach of returns and/or mortality. In this overview paper, we will restrict to the subset of these papers where comonotonicity comes in.

In Koch and De Schepper (2007), stochastic discounting factors are introduced by considering truncated stochastic returns. Analytical results for comonotonic bounds of the present value function of a sum of discounted deterministic cash-flows are derived.

Darkiewicz et al. (2009) first investigate lower and upper bounds for right tails (stop-loss premiums) of deterministic and stochastic sums of non-independent random variables, using the concepts of Sections 1 and 2. Then, the performance of the presented approximations is investigated numerically for individual life annuity contracts as well as for life annuity portfolios. The investment returns are modeled by a Brownian motion process while the mortality is modeled by Makeham's law.

Hoedemakers et al. (2005) and Ahcan et al. (2006) study the distribution of a life annuity (and a portfolio of life annuities) under stochastic interest rates. They apply (4) and (9) for scalar products of mutually independent random vectors and obtain reliable approximations of the underlying distribution functions, in particular very accurate estimates of upper quantiles and stop-loss premiums.

Zhang et al. (2006) consider a homogeneous portfolio composed of $n$ whole-life insurance policies. Since an average insurer usually has a large number of homogeneous policies, they explore the limiting properties of the convex upper bounds of the present value function of such a portfolio. These upper bounds are derived by the technique of comonotonicity under certain assumptions on the dependence structure of the residual lifes of the insured (i.e. independence, positive association, or negative association). The upper bounds are very informative and useful to the insurer in making conservative estimates about the risks and calculating premiums.

Denuit and Dhaene (2007) and Denuit (2007, 2008, 2009) adopt the standard Lee-Carter model for mortality projection when studying portfolios of life annuities. In these papers the discount factors are assumed to be deterministic. In the Lee-Carter model, survival probabilities depend on the future trajectory of the time index, which implies that they become random variables. In the first paper, the concept of comonotonicity is applied to obtain accurate approximations for the stochastic survival probabilities. In Denuit (2008) comonotonicity-based approximations are derived for the quantiles of the conditional expected present value of the annuity payments, given the future path of the Lee-Carter time index.

Denuit, Devolder and Goderniaux suggest in Denuit et al. (2007) securitization of longevity risk in order to offer opportunities for hedging. In particular, they propose the design of survivor bonds which could be issued directly by insurers. In order to guarantee some transparency in the product, the survivor bond is based on a public mortality index. Also here the classical Lee-Carter model for mortality forecasting is used to price a risky coupon survivor bond based 
on this index. The proposed pricing mechanism consists of determining the Wang risk measure of the mortality index which equals the exponential of a linear combination of correlated lognormal random variables. Taking into account comonotonic upper and lower bounds, approximate results are derived.

Spreeuw (2006) applies the theory of comonotonic risks to disability annuities in a Markov model with three states (death, healthy and disabled), where recovery from disabled to health is possible. Benefits are payable during disability whilst premiums are only due whenever the insured is healthy. Starting from the convex upper bound (4) and the improved upper bound (8), he derives two accurate approximations for the sum of the deterministically discounted value of cash-flows involved in such a contract.

\section{Conclusion}

In this paper, we gave an extensive - but not exhaustive - overview of the literature on the theory of comonotonicity and its applications in finance and risk theory, with an emphasis on the literature since 2004 .

Taking into account the huge recent literature on this topic, we may conclude that the concept of comonotonicity indeed plays the role of a helpful tool for solving several research and practical problems in the domain of finance and insurance. It seems very reasonable to assume that the theory of comonotonicity is still in development. This observation makes us believe that in the near future more applications will follow.

In this paper we restricted the applications to financial, actuarial and risk management problems. Without any doubt, the concept of comonotonicity may also be a helpful tool in other domains. An example is the design of wind energy distributed power systems. The problem of defining the dependence structures in the system is tackled by modeling the statistically extreme interdependencies in the system inputs using comonotonicity theory, see, e.g., Papaefthymiou et al. (2004) and Papaefthymiou et al. (2006).

\section{Acknowledgements}

Jan Dhaene acknowledges the financial support of the Onderzoeksfonds K.U. Leuven (GOA/07: Risk Modeling and Valuation of Insurance and Financial Cash Flows, with Applications to Pricing, Provisioning and Solvency).

\section{References}

C. Acerbi. Spectral measures of risk: A coherent representation of subjective risk aversion. Journal of Banking and Finance, 26(7):1505-1518, 2002.

A. Ahcan, G. Darkiewicz, M. Goovaerts, and T. Hoedemakers. Computation of convex bounds for present value functions with random payments. Journal of Computational and Applied Mathematics, 186(1):23-42, 2006.

H. Albrecher, J. Dhaene, M. Goovaerts, and W. Schoutens. Static hedging of Asian options under Lévy models: the comonotonicity approach. The Journal of Derivatives, 12(3):63-72, 2005 .

P. Artzner. Application of coherent capital requirements. North American Actuarial Journal, 3 (2):11-25, 1999. 
P. Artzner, F. Delbaen, J.M. Eber, and D. Heath. Coherent measures of risk. Mathematical Finance, 9:203-228, 1999.

N. Bäuerle and A. Müller. Modeling and comparing dependencies in multivariate risk portfolios. ASTIN Bulletin, 28:59-76, 1998.

P. Boyle and A. Potapchik. Prices and sensitivities of Asian options: A survey. Insurance: Mathematics and Economics, 42(1):189-211, 2008.

K. Brückner. Quantifying the error of convex order bounds for truncated first moments. Insurance: Mathematics and Economics, 42(1):261-270, 2008.

H. Bühlmann. Mathematical Methods in Risk Theory. Springer, Berlin, 1970.

X. Burtschell, J. Gregory, and J.-P. Laurent. A comparative analysis of CDO pricing models under the factor copula framework. Journal of Derivatives, 16(4):9-37, 2009.

P. Chalasani and A. Varikooty. Accurate approximations for European-style Asian options. Journal of Computational Finance, 1(4):11-30, 1998.

X. Chen, G. Deelstra, J. Dhaene, and M. Vanmaele. Static super-replicating strategies for a class of exotic options. Insurance: Mathematics and Economics, 42(3):1067-1085, 2008.

X. Chen, G. Deelstra, J. Dhaene, and M. Vanmaele. Static super-replicating strategies for a class of exotic options: some new developments. Technical report, K.U.Leuven, 2009.

K.C. Cheung. Optimal portfolio problem with unknown dependency structure. Insurance: Mathematics and Economics, 38(1):167-175, 2006.

K.C. Cheung. Optimal allocation of policy limits and deductibles. Insurance: Mathematics and Economics, 41(3):382-391, 2007a.

K.C. Cheung. Characterizations of conditional comonotonicity. Journal of Applied Probability, 44(3):607-617, 2007b.

K.C. Cheung. Improved convex upper bound via conditional comonotonicity. Insurance: Mathematics and Economics, 42(2):651-655, 2008a.

K.C. Cheung. Characterization of comonotonicity using convex order. Insurance: Mathematics and Economics, 43(3):403-406, 2008b.

K.C. Cheung. Upper comonotonicity. Insurance: Mathematics and Economics, 45(1):35-40, 2009.

K.C. Cheung and H. Yang. Ordering of optimal portfolio allocations in a model with a mixture of fundamental risks. Journal of Applied Probability, 45(1):55-66, 2008.

G. Darkiewicz, G. Deelstra, J. Dhaene, T. Hoedemakers, and M. Vanmaele. Bounds for right tails of deterministic and stochastic sums of random variables. Journal of Risk and Insurance, 76(4):847-866, 2009.

G. Deelstra, J. Liinev, and M. Vanmaele. Pricing of arithmetic basket options by conditioning. Insurance: Mathematics and Economics, 34(1):55-77, 2004.

G. Deelstra, I. Diallo, and M. Vanmaele. Bounds for Asian basket options. Journal of Computational and Applied Mathematics, 218(2):215-228, 2008. 
G. Deelstra, I. Diallo, and M. Vanmaele. Moment matching approximation of Asian basket option prices. Journal of Computational and Applied Mathematics, 2009a. doi:10.1016/j.cam.2009.03.004.

G. Deelstra, A. Petkovic, and M. Vanmaele. Pricing and hedging Asian basket spread options. Journal of Computational and Applied Mathematics, 2009b. doi:10.1016/j.cam.2009.11.027.

G. Deelstra, M. Vanmaele, and D. Vyncke. Minimizing the risk of a financial product using a put option. Journal of Risk and Insurance, 2010. Accepted.

D. Denneberg. Non-Additive Measure and Integral. Kluwer Academic Publishers, Boston, 1994.

M. Denuit. Distribution of the random future life expectancies in log-bilinear mortality projection models. Lifetime Data Analysis, 13(3):381-397, 2007.

M. Denuit. Comonotonic approximations to quantiles of life annuity conditional expected present value. Insurance: Mathematics and Economics, 42(2):831-838, 2008.

M. Denuit. Life anuities with stochastic survival probabilities: A review. Methodology and Computing in Applied Probability, 11(3, Sp. Iss. SI):463-489, 2009.

M. Denuit and J. Dhaene. Simple characterizations of comonotonicity and countermonotonicity by extremal correlations. Belgian Actuarial Bulletin, 3:22-27, 2003.

M. Denuit and J. Dhaene. Comonotonic bounds on the survival probabilities in the Lee-Carter model for mortality projection. Journal of Computational and Applied Mathematics, 203(1): 169-176, 2007.

M. Denuit, J. Dhaene, and C. Ribas. Does positive dependence between individual risks increase stop-loss premiums? Insurance: Mathematics and Economics, 28(3):305-308, 2001.

M. Denuit, J. Dhaene, M. Goovaerts, and R. Kaas. Actuarial theory for dependent risks: measures, orders and models. Wiley, 2005.

M. Denuit, P. Devolder, and A.-C. Goderniaux. Securitization of longevity risk: Pricing survivor bonds with Wang transform in the Lee-Carter framework. Journal of Risk and Insurance, 74 (1):87-113, 2007.

J. Dhaene and M. Denuit. The safest dependency structure among risks. Insurance: Mathematics and Economics, 25(1):11-21, 1999.

J. Dhaene and M. Goovaerts. Dependency of risks and stop-loss order. ASTIN Bulletin, 26(2): 201-212, 1996.

J. Dhaene and M. Goovaerts. On the dependency of risks in the individual life model. Insurance: Mathematics and Economics, 19(3):243-253, 1997.

J. Dhaene, S.S. Wang, V.R. Young, and M.J. Goovaerts. Comonotonicity and maximal stop-loss premiums. Bulletin of the Swiss Association of Actuaries, 2:99-113, 2000.

J. Dhaene, M. Denuit, M.J. Goovaerts, R. Kaas, and D. Vyncke. The concept of comonotonicity in actuarial science and finance: Theory. Insurance: Mathematics and Economics, 31(1):3-33, $2002 \mathrm{a}$.

J. Dhaene, M. Denuit, M.J. Goovaerts, R. Kaas, and D. Vyncke. The concept of comonotonicity in actuarial science and finance: Application. Insurance: Mathematics and Economics, 31(2): 133-161, 2002b. 
J. Dhaene, M. Goovaerts, and R. Kaas. Economic capital allocation derived from risk measures. North American Actuarial Journal, 7(2):44-59, 2003.

J. Dhaene, S. Vanduffel, M.J. Goovaerts, R. Kaas, and D. Vyncke. Comonotonic approximations for optimal portfolio selection problems. Journal of Risk of Insurance, 72(2):253-300, 2005.

J. Dhaene, A. Kukush, and M. Pupashenko. On the structure of premium principles under pointwise comonotonicity. Theory of Stochastic Processes, 12(28, N3-4):27-45, 2006a.

J. Dhaene, S. Vanduffel, M.J. Goovaerts, R. Kaas, Q. Tang, and D. Vyncke. Risk measures and comonotonicity: A review. Stochastic Models, 22(4):573-606, 2006b.

J. Dhaene, L. Henrard, Z. Landsman, A. Vandendorpe, and S. Vanduffel. Some results on the CTE-based capital allocation rule. Insurance: Mathematics and Economics, 42(2):855-863, 2008a.

J. Dhaene, R.J.A. Laeven, S. Vanduffel, G. Darkiewicz, and M.J. Goovaerts. Can a coherent risk measure be too subadditive? Journal of Risk and Insurance, 75(2):365-386, 2008b.

J. Dhaene, M. Denuit, and S. Vanduffel. Correlation order, merging and diversification. Insurance: Mathematics and Economics, 45(3):325-332, 2009a.

J. Dhaene, A. Tsanakas, E. Valdez, and S. Vanduffel. Optimal capital allocation principles. Technical report, K.U.Leuven, 2009b.

I. Ekeland, A. Galichon, and M. Henry. Comonotonic measures of multivariate risks. Technical Report 25, Ecole Polytechnique/Centre National de la Recherche Scientifique, 2009.

P. Embrechts, A. Mc Neil, and D. Straumann. Correlation and dependency in risk management: properties and pitfalls. In M. Dempster and H.K. Moffat, editors, Risk Management: Valueat-Risk and Beyond, pages 176-223. Cambridge University Press, 2002.

P. Embrechts, A. Höing, and G. Puccetti. Worst VaR scenarios. Insurance: Mathematics and Economics, 37(1):115-134, 2005.

M. Fréchet. Sur les tableaux de correlation dont les marges sont donnés. Annales de l'Université de Lyon Sect.A, 14:53-77, 1951.

A. Galichon and M. Henry. Dual theory of choice under multivariate risks. Technical report, SSRN eLibrary, 2008.

K. Glau, N. Vandaele, and M. Vanmaele. Hedging interest rate derivatives in a Lévy driven term structure model. Technical report, Ghent University, 2009.

M. Goovaerts and J. Dhaene. Supermodular ordering and stochastic annuities. Insurance: Mathematics and Economics, 24(3):281-290, 1999.

M. Goovaerts, R. Kaas, R. Laeven, and Q. Tang. A comonotonic image of independence for additive risk measures. Insurance: Mathematics and Economics, 35(3):581-594, 2004.

W.R. Heilmann. On the impact of the independence of risks on stop-loss premiums. Insurance: Mathematics and Economics, 5(3):197-199, 1986.

D. Hobson, P. Laurence, and T.H. Wang. Static-arbitrage upper bounds for the prices of basket options. Quantitative Finance, 5(4):329-342, 2005 a. 
D. Hobson, P. Laurence, and T.H. Wang. Static-arbitrage optimal subreplicating strategies for basket options. Insurance: Mathematics and Economics, 37(3):553-572, 2005b.

T. Hoedemakers, G. Darkiewicz, and M. Goovaerts. Approximations for life annuity contracts in a stochastic financial environment. Insurance: Mathematics and Economics, 37(2):239-269, 2005.

W. Hoeffding. Masstabinvariante Korrelationstheorie. Schriften des mathematischen Instituts und des Instituts für angewandte Mathematik des Universitat Berlin, 5:179-233, 1940.

L. Hua and K.C. Cheung. Stochastic orders of scalar products with applications. Insurance: Mathematics and Economics, 42(3):865-872, 2008a.

L. Hua and K.C. Cheung. Worst allocations of policy limits and deductibles. Insurance: Mathematics and Economics, 43(1):93-98, 2008b.

P.J. Huber. Robust Statistics. Wiley, New York, 1981.

F. Jamshidian. An exact bond option formula. Journal of Finance, XLIV(1):205-209, 1989.

H. Joe. Multivariate Models and Dependence Concepts. Chapman \& Hall, London, 1997.

E. Jouini and C. Napp. Conditional comonotonicity. Decisions in Economics and Finance, 27 (2):153-166, 2004.

E. Jouini, W. Schachermayer, and N. Touzi. Optimal risk sharing for law invariant monetary utility functions. Mathematical Finance, 18(2):269-292, 2008.

R. Kaas, J. Dhaene, and M. Goovaerts. Upper and lower bounds for sums of random variables. Insurance: Mathematics and Economics, 27(2):151-168, 2000.

R. Kaas, J. Dhaene, D. Vyncke, M. Goovaerts, and M. Denuit. A simple geometric proof that comonotonic risks have the convex-largest sum. ASTIN Bulletin, 32(1):71-80, 2002.

I. Koch and A. De Schepper. An application of comonotonicity and convex ordering to present values with truncated stochastic interest rates. Insurance: Mathematics and Economics, 40 (3):386-402, 2007.

A. Kukush and M. Pupashenko. Bounds for a sum of random variables under a mixture of normals. Theory of Stochastic Processes, 13(29, N4):82-97, 2007.

R.J.A. Laeven. Worst VaR scenarios: A remark. Insurance: Mathematics and Economics, 44 (2, Sp. Iss. SI):159-163, 2009.

R.J.A. Laeven and M.J. Goovaerts. An optimization approach to the dynamic allocation of economic capital. Insurance: Mathematics and Economics, 35(2):299-319, 2004.

P. Laurence and T.-H. Wang. Distribution-free upper bounds for spread options and marketimplied antimonotonicity gap. European Journal of Finance, 14(8):717-734, 2008.

P. Laurence and T.-H. Wang. Sharp distribution free lower bounds for spread options and the corresponding optimal subreplicating portfolios. Insurance: Mathematics and Economics, 44 (1):35-47, 2009.

R. Lord. Partially exact and bounded approximations for arithmetic Asian options. Journal of Computational Finance, 10(2):1-52, 2006. 
M. Ludkovski and L. Rüschendorf. On comonotonicity of Pareto optimal risk sharing. Statistics ES Probability Letters, 78(10):1181-1188, 2008.

I. Meilijson and A. Nadas. Convex majorization with an application to the length of critical paths. Journal of Applied Probability, 16(3):671-676, 1979.

A. Müller. Stop-loss order for portfolios of dependent risks. Insurance: Mathematics and Economics, 21(2):219-223, 1997.

R.B. Nelsen. An Introduction to Copulas. Number 139 in Lecture Notes in Statistics. Springer Verlag, New York, 1998.

J.A. Nielsen and K. Sandmann. Pricing bounds on Asian options. Journal of Financial and Quantitative Analysis, 38(2):449-473, 2003.

G. Papaefthymiou, P.H. Schavemaker, L. van der Sluis, and A. Tsanakas. Design of wind energy distributed power systems: Investigation of stochastic bounds using Monte Carlo simulation. In P.D. Bourkas and P. Halaris, editors, Proceedings of the Fourth IASTED International Conference on Power and Energy Systems, pages 115-121, 2004.

G. Papaefthymiou, P.H. Schavemaker, L. van der Sluis, W.L. Kling, D. Kurowicka, and R.M. Cooke. Integration of stochastic generation in power systems. International Journal of Electrical Power \& Energy Systems, 28(9):655-667, 2006.

G. Puccetti and M. Scarsini. Multivariate comonotonicity. Journal of Multivariate Analysis, 101(1):291-304, 2010.

H. Reynaerts, M. Vanmaele, J. Dhaene, and G. Deelstra. Bounds for the price of a Europeanstyle Asian option in a binary tree model. European Journal of Operational Research, 168(2): 322-332, 2006.

A. Roëll. Risk aversion in Quiggin and Yaari's rank-order model of choice under uncertainty. The Economic Journal, 97(133a):143-159, 1987.

L.C.G. Rogers and Z. Shi. The value of an Asian option. Journal of Applied Probability, 32(4): 1077-1088, 1995.

L. Rüschendorf. Solution of statistical optimization problem by rearrangement methods. Metrika, 30:55-61, 1983.

D. Schmeidler. Integral representation without additivity. Proceedings of the American Mathematical Society, 97:255-261, 1986.

D.F. Schrager and A.A.J. Pelsser. Pricing rate of return guarantees in regular premium unit linked insurance. Insurance: Mathematics and Economics, 35(2):369-398, 2004.

M. Shaked and J.G. Shanthikumar. Stochastic Orders and their Applications. Academic Press, New York, 1994.

Q. Shang, X. Qin, and Y. Wang. Design of catastrophe mortality bonds based on the comonotonicity theory and jump-diffusion process. International Journal of Innovative Computing Information and Control, 5(4):991-1000, 2009.

S. Simon, M.J. Goovaerts, and J. Dhaene. An easy computable upper bound for the price of an arithmetic Asian option. Insurance: Mathematics and Economics, 26(2-3):175-184, 2000. 
J. Spreeuw. Two approximations of the present value distribution of a disability annuity. Journal of Computational and Applied Mathematics, 186(1):217-231, 2006.

A. Tchen. Inequalities for distributions with given marginals. The Annals of Probability, 8(4): 814-827, 1980.

G. Thompson. Fast narrow bounds on the value of Asian options. Technical report, University of Cambridge, 1999.

A. Tsanakas. Risk measurement in the presence of background risk. Insurance: Mathematics and Economics, 42(2):520-528, 2008.

A. Tsanakas. To split or not to split: Capital allocation with convex risk measures. Insurance: Mathematics and Economics, 44(2, Sp. Iss. SI):268-277, 2009.

A. Tsanakas and N. Christofides. Risk exchange with distorted probabilities. ASTIN Bulletin, 36(1):219-243, 2006.

E.A. Valdez, J. Dhaene, M. Maj, and S. Vanduffel. Bounds and approximations for sums of dependent log-elliptical random variables. Insurance: Mathematics and Economics, 44(3): 385-397, 2009.

S. Vanduffel, J. Dhaene, M. Goovaerts, and R. Kaas. The hurdle-race problem. Insurance: Mathematics and Economics, 33(2):405-413, 2003.

S. Vanduffel, J. Dhaene, and M. Goovaerts. On the evaluation of 'saving-consumption' plans. Journal of Pension Economics and Finance, 4(1):17-30, 2005a.

S. Vanduffel, T. Hoedemakers, and J. Dhaene. Comparing approximations for risk measures of sums of non-independent lognormal random variables. North American Actuarial Journal, 9 (4):71-82, 2005b.

S. Vanduffel, X. Chen, J. Dhaene, M. Goovaerts, L. Henrard, and R. Kaas. Optimal approximations for risk measures of sums of lognormals based on conditional expectations. Journal of Computational and Applied Mathematics, 221(1):202-218, 2008a.

S. Vanduffel, Z. Shang, L. Henrard, J. Dhaene, and E.A. Valdez. Analytic bounds and approximations for annuities and Asian options. Insurance: Mathematics and Economics, 42(3): 1109-1117, 2008b.

M. Vanmaele, G. Deelstra, and J. Liinev. Approximation of stop-loss premiums involving sums of lognormals by conditioning on two variables. Insurance: Mathematics and Economics, 35 (2):343-367, 2004.

M. Vanmaele, G. Deelstra, J. Liinev, J. Dhaene, and M. Goovaerts. Bounds for the price of discrete arithmetic Asian options. Journal of Computational and Applied Mathematics, 185 (1):51-90, 2006.

O. Vasicek. An equilibrium characterisation of the term structure. Journal of Financial Economics, 5:177-188, 1977.

J. von Neumann and O. Morgenstern. Theory of Games and Economic Behavior. Princeton University Press, Princeton, second edition, 1947.

D. Vyncke, M. Goovaerts, and J. Dhaene. An accurate analytical approximation for the price of a European-style arithmetic Asian option. Finance, 25:121-139, 2004. 
S. Wang and J. Dhaene. Comonotonicity, correlation order and premium principles. Insurance: Mathematics and Economics, 22(3):235-242, 1998.

S.S. Wang and V.R. Young. Ordering risks: expected utility theory versus Yaari's dual theory of risk. Insurance: Mathematics and Economics, 22(2):145-162, 1998.

$\mathrm{X}$. Wu and X. Zhou. A new characterization of distortion premiums via countable additivity for comonotonic risks. Insurance: Mathematics and Economics, 38(2):324-334, 2006.

M.E. Yaari. The dual theory of choice under risk. Econometrica, 55(1):95-115, 1987.

J. Yang, S. Cheng, and L. Zhang. Bivariate copula decomposition in terms of comonotonicity, countermonotonicity and independence. Insurance: Mathematics and Economics, 39(2):267$284,2006$.

Y. Zhang, Z. Lin, and C. Weng. Some limiting properties of the bounds of the present value function of a life insurance portfolio. Journal of Applied Probability, 43(4):1155-1164, 2006.

W. Zhuang, Z. Chen, and T. Hu. Optimal allocation of policy limits and deductibles under distortion risk measures. Insurance: Mathematics and Economics, 44(3):409-414, 2009. 